\title{
Frequency Doubling Technology Perimetry and Central Corneal Thickness in people with high intraocular pressures and normal discs
}

\author{
Dr Subhrangshu Sengupta, Prof Gautam Bhaduri, Prof Himadri Datta \\ Regional Institute of Ophthalmology, Medical College \& Hospital, Kolkata - 700073. West Bengal. India.
}

\begin{abstract}
Aim: To study the correlation between central corneal thickness (CCT) measured by ultrasound pachymetry and Frequency Doubling Technology Perimetry (FDT) in patients aged more than or equal to 40 years with high IOP and normal discs.

Materials and Methods: This research work has two arms, the rural arm is an eye care centre in Hooghly District of West Bengal (Village: Kuliapara, P.O. Dhobapara, P.S. Balagarh)and the urban arm is a tertiary eye hospital in Kolkata, West Bengal. People aged 40 years and above or those turning 40 in the calendar year with high IOP and normal discs and not on any topical or systemic medications underwent complete ophthalmological examination including CCT assessment by ultrasound pachymetry (Ocuscan RxP) and FDT (Humphrey, Carl Zeiss). Patients with history of intraocular surgery, ocular trauma, corneal opacities preventing accurate applanation tonometry and media haze dense enough to preclude adequate fundal view were not enumerated.

Results: One hundred and eighty subjects fulfilled the laid down study inclusion criteria. Average CCT for the right eye $(R E)$ is $530.74 \mu$ and that for the left eye $(L E)$ is $531.32 \mu(p=0.61$, not statistically significant). IOP in the RE varied from 18 to $32 \mathrm{~mm} \mathrm{Hg}$ with an average value of $23 \mathrm{~mm} \mathrm{Hg}$ and that in the LE varied from 14 to 32 $\mathrm{mm} \mathrm{Hg}$ with an average value of $22 \mathrm{~mm} \mathrm{Hg}$. Abnormal FDT in either eye was present in eighteen subjects of whom nine subjects had bilateral abnormal FDT and eight subjects had only RE affection and one subject had only LE affection.

Conclusion: Our study concludes that the included subjects with abnormal FDT had thinner CCTs as compared to those with a normal FDT. Extending this relation further, we conclude that among subjects with a higher IOP and normal discs or patients grouped as ocular hypertensives, thicker corneas lower the risk for development of early glaucomatous functional damage as picked up by FDT, as compared to patients with thinner corneas.
\end{abstract}

\section{Introduction}

Glaucoma is an optic neuropathy characterized by progressive loss of retinal ganglion cells, which ultimately can lead to functional loss, visual disability, and blindness. ${ }^{1}$

Intraocular pressure (IOP) is a modifiable risk factor for glaucoma. IOP may be underestimated in eyes with thin corneas and overestimated in eyes with thick corneas. ${ }^{2-5}$

A report of the landmark Ocular Hypertension Treatment Study(OHTS) showed that central corneal thickness (CCT) was found to be a powerful predictor of development of primary open-angle glaucoma among ocular hypertensive eyes. ${ }^{6}$ Eyes with CCT of $555 \mu \mathrm{m}$ or less had a threefold greater risk of glaucoma developing than participants who had CCT of more than $588 \mu \mathrm{m}$.

Frequency doubling technology (FDT) perimetry has been proposed as a test for the early detection of glaucomatous functional damage. ${ }^{7,8}$ Testing involves presentation of frequency-doubling stimulus, and the contrast sensitivity of the stimulus is adjusted to determine the limit of detection. Several independent studies have shown that FDT has high sensitivity and specificity for discriminating glaucomatous and healthy subjects. ${ }^{7}$ This present study aims to study the correlation between CCT measured by ultrasound pachymetry and FDT in patients aged more than or equal to 40 years with high IOP and normal discs.

\section{Materials and Methods:}

Institutional Ethics Committee clearance was obtained for conducting this population based cross sectional study. This research work has two arms, the rural arm is an eye care centre in Hooghly District of West Bengal (Village: Kuliapara, P.O. Dhobapara, P.S. Balagarh)and the urban arm is a tertiary eye hospital in Kolkata, West Bengal.

People aged 40 years and above or those turning 40 in the calendar year and not on any topical or systemic medications were examined. Patients with history of intraocular surgery, ocular trauma, corneal opacities preventing accurate applanation tonometry and media haze dense enough to preclude adequate fundal view were not enumerated. 


\section{All patients were examined as follows :}

(a) Ocular and medical history -- A detailed history pertaining to medical and ophthalmic problems was elicited. Data collected includes use of glasses and its duration, history of previous trauma or surgery or laser in the eyes, history of ocular and oral medication, significant systemic illness with special reference to diabetes or hypertension, significant family history and addiction history.

(b) Refraction and recording of uncorrected and best-corrected visual acuity

(c) Torch light external ocular examination including ocular movements and any other obvious strabismus / eyelid pathology.

(d) Pupillary evaluation - Carried out in dim light conditions. The patient is asked to focus on a distant object and the strength and the direct and indirect reaction of each pupil noted. The presence or absence of an afferent pupillary defect is also checked for.

(e) Slit lamp biomicroscopy, including Van Herick grading of the angle of the anterior chamber angle.

(f) Applanation tonometry using the Goldmann Applanation Tonometer .

(g)Corneal pachymetry - Central corneal thickness using ultrasound pachymetry (Ocuscan RxP, Alcon labs, USA).Average of three readings, rounded off to the nearest whole number, was taken.

(h)Gonioscopy - Done in dim illumination using a narrow slit beam not extending onto the pupillary area. Goldmann single mirror lens is used for the purpose. The angle is graded using Shaffer's grading system.

(i) Grading of lens opacities - using the LOCS II Classification after pupillary dilatation.

(k) Evaluation of optic disc using +78D lens. The vertical cup-disc ratio (VCDR) was recorded and any other significant findings like presence of peri papillary atrophy, optic disc/ peripapillary hemorrhage, bayoneting sign, baring of circumlinear vessels, laminar dot sign, etc were recorded.

(1)Frequency Doubling Perimetry(Humphrey FDT, Carl Zeiss, Dublin, USA) was performed in all patients. FDT perimetry was performed using full-threshold program N-30 (Welch Allyn FDT, Skaneateles Falls, NY). In this test, target stimuli consist of individual $10^{\circ}$ square, $0.25 \mathrm{cyc} /{ }^{\circ}$ sinusoidal gratings, counterphasing at $25 \mathrm{~Hz}$. Targets are presented in 1 of 19 test areas located within the central $30^{\circ}$ of the visual field. The test uses a modified binary search staircase threshold procedure with stimuli presented for a maximum of $720 \mathrm{msec}$ and measures the contrast needed for detection of the stimulus. During the first $160 \mathrm{msec}$, stimulus contrast is increased gradually from zero to the contrast selected for that presentation. If the stimulus is not seen, it remains at this contrast for up to $400 \mathrm{msec}$ and then is gradually decreased to zero during the final $160 \mathrm{msec}$. The interstimulus interval varies randomly up to $500 \mathrm{msec} .{ }^{9}$ Abnormality for an FDT examination was determined by comparison with the manufacturer's internal normative database. An abnormal examination was defined for this study by the presence of at least two test areas (points) with $\mathrm{P}<0.05$ or worse on the pattern deviation plot or by the presence of a PSD with $\mathrm{P}<0.05$ or worse. To be included, all subjects had to have reliable visual field results. This was defined as $25 \%$ or fewer false-positive results, false-negative results, and fixation losses.

All subjects over 40 years or turning 40 in the calendar year and gonioscopy revealing Shaffer's Grading 3 or more in more than 180 degrees in both eyes were included for enumeration and analysis. More than two thousand people were thus enumerated.

All of the enumerated patients with IOP more than or equal to $21 \mathrm{~mm} \mathrm{Hg}$ in either eye or VCDR of less than or equal to 0.5 in both the eyes were included for this current study.

\section{Results :}

One hundred and eighty subjects fulfilled the laid down study inclusion criteria. Average age of the subjects was 55 years and male : female ratio is 85:95. Average CCT for the right eye (RE) is $530.74 \mu$ and that for the left eye (LE) is $531.32 \mu(\mathrm{p}=0.61)$, not statistically significant). IOP in the RE varied from 18 to $32 \mathrm{~mm}$ $\mathrm{Hg}$ with an average value of $23 \mathrm{~mm} \mathrm{Hg}$ and that in the LE varied from 14 to $32 \mathrm{~mm} \mathrm{Hg}$ with an average value of $22 \mathrm{~mm} \mathrm{Hg}$. However, interestingly there is a statistically significant difference in the average IOP values of the two eyes $(\mathrm{p}<0.001)$. Abnormal FDT in either eye (defined by the presence of at least two test areas (points) with $\mathrm{P}<0.05$ or worse on the pattern deviation plot or by the presence of a PSD with $\mathrm{P}<0.05$ or worse) was present in eighteen subjects of whom nine subjects had bilateral abnormal FDT and eight subjects had only RE affection and one subject had only LE affection. The CCT values (rounded to the nearest whole number) obtained by ultrasound pachymetry in the various groups are detailed in the table below :

\begin{tabular}{|l|l|l|l|l|}
\hline Groups & Number of subjects & CCT in Right Eye $(\mu)$ & CCT in Left Eye $(\mu)$ & Average CCT $(\mu)$ \\
\hline Bilateral abnormal FDT & 9 & $516 \mu$ & $518 \mu$ & $517 \mu$ \\
\hline $\begin{array}{l}\text { Only Right Eye Abnormal } \\
\text { FDT }\end{array}$ & 8 & $521 \mu$ & $531 \mu$ & $526 \mu$ \\
\hline $\begin{array}{l}\text { Only Left Eye Abnormal } \\
\text { FDT }\end{array}$ & 1 & $477 \mu$ & $472 \mu$ & $475 \mu$ \\
\hline $\begin{array}{l}\text { Abnormal FDT in one or } \\
\text { both eyes }\end{array}$ & 18 & $516 \mu$ & $521 \mu$ & $519 \mu$ \\
\hline Normal FDT in both eyes & 162 & $532 \mu$ & $533 \mu$ & $532 \mu$ \\
\hline
\end{tabular}




\section{Discussions and Conclusion :}

In our study, all included subjects had high IOP with normal VCR and in the absence of standard automated perimetry (SAP) results, can be referred to as probable ocular hypertensives (OHT). Higher number of females in this group as compared to males is similar to various other similar studies. ${ }^{9}$

With high sensitivity and specificity for glaucoma diagnosis, FDT perimetry is increasingly used as a diagnostic tool for glaucoma ${ }^{10,11}$ FDT perimetry also offers the potential advantage of reduced testing time and less variability compared with (SAP). ${ }^{12}$ Overall $10 \%(18 / 180)$ of the subjects had abnormal FDT in either or both eyes. This is in accordance with previous studies in which the prevalence of FDT abnormalities in eyes with OHT has been generally reported to be between $11 \%$ and $46 \%$, depending on the criteria used and the specific risk characteristics of the population evaluated. ${ }^{12,13}$

With the screening protocol of FDT, Quigley ${ }^{14}$ found that the criterion with best performance to discriminate between glaucoma and normal eyes was the presence of two or more abnormal locations, regardless of the severity of the defect. This criterion resulted in a sensitivity of $91 \%$ with specificity of $94 \%$. In his study, Quigley also found that the severity of abnormal points with FDT was not as well correlated with glaucoma damage as the total number of abnormal points

Among the eyes included in our study, average CCT of both the eyes as well as individual eyes was lower in the abnormal FDT group as compared to the subjects with normal FDT in both eyes. This difference is not statistically significant when the individual eyes are compared in the two groups (for $\mathrm{RE} p=0.055$ and for LE $\mathrm{p}=0.13$ ). However when the average CCT for both eyes are compared between the two groups of with normal and abnormal FDT, a statistically significant difference is obtained $(\mathrm{p}=0.013$, ie $\mathrm{p}<0.05)$. This result is similar to that obtained by Medeiros et $\mathrm{al}^{9}$ who studied 65 eyes with OHT and normal optic discs. They found that patients currently diagnosed with OHT, but with visual field loss detected by FDT perimetry, had significantly lower CCT measurements than patients with OHT with normal FDT results.

FDT is widely accepted as a test for early detection of functional glaucomatous damage. Our study concludes that the included subjects with abnormal FDT had thinner CCTs as compared to those with a normal FDT. Extending this relation further, we conclude that among subjects with a higher IOP and normal discs or patients grouped as ocular hypertensives, thicker corneas lower the risk for development of early glaucomatous functional damage as picked up by FDT, as compared with patients with thinner corneas.

This study focuses on the subgroup of patients with normal discs and high IOP. Generally a normal SAP for this group classifies them as ocular hypertensives and they are mainly followed up without initiation of anti glaucoma drugs. However, if these patients are not adequately motivated and explained regarding their ocular condition with special emphasis on the need for follow up at regular intervals, there is a very high chance of them being lost to follow up. The findings of our study further substantiates the need for very close follow up in those who have a thinner CCT, by showing the appearance of functional glaucomatous damage in this group through FDT. Further this study also assumes significance in the setting of rural India where a Humphrey Standard Automated Perimeter may not be easily accessible. FDT being a portable instrument, our study shows that this instrument can be used effectively to stratify the sub group of OHTs with lower CCT into a very important target group for regular follow up. This can further help in the better resource and manpower utilisation. Therefore this study establishes that CCT should be taken into account when assessing the risk of patients with OHT to develop glaucoma.

\section{Acknowledgement :} Bengal. India

The West Bengal University of Health Sciences, DD-36, Salt Lake, Sector -1, Kolkata 700064.West

\section{References :}

[1]. Weinreb RN, Khaw PT. Primary open-angle glaucoma. Lancet 2004;363:1711-20.

[2]. Johnson M, Kass MA, Moses RA, Grodzki WJ. Increased corneal thickness simulating elevated intraocular pressure. Arch Ophthalmol 1978;96:664-5.

[3]. Brandt JD. The influence of corneal thickness on the diagnosis and management of glaucoma. J Glaucoma 2001;10(5 Suppl1):S657 .

[4]. Stodtmeister R. Applanation tonometry and correction according to corneal thickness. Acta Ophthalmol Scand 1998;76:319-24.

[5]. Doughty MJ, Zaman ML. Human corneal thickness and its impact on intraocular pressure measures: a review and metaanalysis approach. Surv Ophthalmol 2000;44:367-408.

[6]. Gordon MO, Beiser JA, Brandt JD, et al. The Ocular Hypertension Treatment Study. baseline factors that predict the onset of primary open-angle glaucoma. Arch Ophthalmol 2002;120:714-20; discussion 829-30.

[7]. Landers J, Goldberg I, Graham S. A comparison of short wavelength automated perimetry with frequency doubling perimetry for the early detection of visual field loss in ocular hypertension. Clin Experiment Ophthalmol 2000;28:248-52.

[8]. Sample PA, Bosworth CF, Blumenthal EZ, et al. Visual function-specific perimetry for indirect comparison of different ganglion cell populations in glaucoma. Invest Ophthalmol Vis Sci 2000;41:1783-90.

[9]. Medeiros FA, Sample PA, Weinreb RN. Corneal Thickness Measurements and Frequency Doubling Technology Perimetry Abnormalities in Ocular Hypertensive Eyes. Ophthalmology 2003;110:1903-1908. 
[10]. Cello KE, Nelson-Quigg JM, Johnson CA. Frequency doubling technology perimetry for detection of glaucomatous visual field loss. Am J Ophthalmol 2000;129:314-22.

[11]. Horn FK, Wakili N, Junemann AM, Korth M. Testing for glaucoma with frequency-doubling perimetry in normals, ocular hypertensives, and glaucoma patients. Graefes Arch Clin Exp Ophthalmol 2002;240:658-65.

[12]. Spry PG, Johnson CA, McKendrick AM, Turpin A. Variability components of standard automated perimetry and frequencydoubling technology perimetry. Invest Ophthalmol Vis Sci 2001;42:1404-10.

[13]. Horn FK, Wakili N, Junemann AM, Korth M. Testing for glaucoma with frequency-doubling perimetry in normals, ocular hypertensives, and glaucoma patients. Graefes Arch ClinExp Ophthalmol 2002;240:658-65.

[14]. Landers J, Goldberg I, Graham S. A comparison of short wavelength automated perimetry with frequency doubling perimetry for the early detection of visual field loss in ocular hypertension. Clin Experiment Ophthalmol 2000;28:248-52.

[15]. Quigley HA. Identification of glaucoma-related visual field abnormality with the screening protocol of frequency doubling technology. Am J Ophthalmol 1998;125:819-29. 